\begin{tabular}{|c|c|c|c|c|c|c|c|c|c|}
\hline Score & $\begin{array}{l}<1 / \\
16\end{array}$ & $\begin{array}{l}1 / \\
16\end{array}$ & $\begin{array}{l}1 / \\
32\end{array}$ & $\begin{array}{l}1 / 64 \\
(+v e)\end{array}$ & $\begin{array}{l}1 / 128 \\
\text { (+ve) }\end{array}$ & $\begin{array}{l}\text { Not } \\
\text { detected } \\
\text { (in urine) }\end{array}$ & $\begin{array}{l}\text { Detected } \\
\text { (in urine) }\end{array}$ & $\begin{array}{l}\text { No result } \\
\text { or } \\
\text { comment }\end{array}$ & Tota \\
\hline CURB 0 & 65 & 5 & 5 & 0 & 0 & 17 & 0 & 12 & 104 \\
\hline CURB 1 & 57 & 5 & 5 & 1 & 0 & 19 & 0 & 9 & 96 \\
\hline CURB 2 & 72 & 13 & 2 & 0 & 0 & 20 & 0 & 13 & 120 \\
\hline CURB 3 & 62 & 12 & 3 & 0 & 0 & 15 & 0 & 11 & 103 \\
\hline CURB 4 & 14 & 0 & 2 & 0 & 0 & 5 & 0 & 3 & 24 \\
\hline CURB 5 & 3 & 0 & 0 & 0 & 0 & 0 & 0 & 0 & 3 \\
\hline $\begin{array}{l}\text { Not } \\
\text { recorded }\end{array}$ & 305 & 49 & 15 & 2 & 1 & 86 & 1 & 61 & 520 \\
\hline $\begin{array}{l}\text { Not in } \\
\text { database }\end{array}$ & 14 & 3 & 0 & 0 & 0 & 2 & 0 & 2 & 21 \\
\hline TOTAL & 592 & 87 & 32 & 3 & 1 & 164 & 1 & 111 & 991 \\
\hline
\end{tabular}

\section{P261 DEVELOPMENT OF AN EXTENDED SPECIFICITY MULTIPLEX IMMUNOASSAY USING HUMAN MONOCLONAL ANTIBODIES FOR DETECTION OF STREPTOCOCCUS PNEUMONIAE SEROTYPE-SPECIFIC ANTIGEN IN URINE}

${ }^{1} \mathrm{~S}$ Eletu, ${ }^{1} \mathrm{C}$ Sheppard, ${ }^{2} \mathrm{E}$ Thomas, ${ }^{3} \mathrm{~K}$ Smith, ${ }^{1} \mathrm{D}$ Litt, ${ }^{1} \mathrm{~N}$ Fry. ${ }^{1}$ Respiratory and Vaccine Preventable Bacterial Reference Unit, London, UK; ${ }^{2}$ Department of Biology, University of Washington, Seattle, USA; ${ }^{3}$ Oklahoma Medical Research Foundation, Oklahoma City, USA

\subsection{6/thoraxinl-2016-209333.404}

Streptococcus pneumoniae (pneumococcus) is a major cause of morbidity and mortality worldwide. To date 93 capsular serotypes of pneumococcus have been described, but many of these are rarely found in disease. Currently vaccines are targeted at between 7 and 23 of the most common circulating serotypes. However, with the uptake of any pneumococcal serotype based vaccine the risk of serotype-replacement and an increase in disease caused by non-vaccine serotypes remains. This highlights the importance of determination of the serotype responsible for the infection.

The diagnosis and subsequent serotype surveillance of pneumococcal infection relies heavily on culture techniques which are known to be insensitive, particularly in cases of non-invasive disease. There are, therefore, potentially many pneumococcal disease cases where an isolate for serotyping is never obtained. Urine antigen detection using methods such as BinaxNOW (Alere) can be used to confirm pneumococcal infection in the absence of an isolate, but does not give serotype information.

Previously described serotype-specific urine assays covering mainly conjugate vaccine serotypes, give no/very little information about circulating non-vaccine serotypes and are currently only available in one or two specialist laboratories.

Our laboratory has just completed initial development of an extended range antigen capture Luminex based assay to detect $S$. pneumoniae serotype specific antigen in urine samples using fully human human, full length monoclonal antibodies. The assay covers 24 different serotypes/groups plus C-polysaccharide, including all the currently available conjugate vaccine and 23 -valent polysaccharide vaccine types plus some cross-reactive serotypes.

We have validated the assay for sensitivity, specificity and reproducibility using spiked urine samples and a panel of BinaxNOW tested clinical urine specimens, some of which were from patients from whom a pneumococcal isolate was also cultured. The results for the validation will be presented.

This assay can be extended to testing other clinical samples such as cerebrospinal and pleural fluids and with development has the potential to greatly improve serotype-specific surveillance in the many cases of pneumococcal disease from which a culture is never obtained.

\section{P262 THE RELATIONSHIP BETWEEN PENETRATION OR ASPIRATION OF ORAL INTAKE AND CHEST INFECTIONS IN ATAXIA TELANGIECTASIA PATIENTS}

CBR Mossey-Gaston, SG Ellum, AC DeSousae, N Oscroft, P Hales, H Baxendale. Papworth Hospital NHS Foundation Trust, Papworth Everard, Cambridge, UK

\subsection{6/thoraxjnl-2016-209333.405}

Introduction and objectives As part of the care of Ataxia Telangiectasia Patients, a baseline swallow profile is obtained using a videofluoroscopy. It was observed that penetration or aspiration of oral intake did not appear to have a direct link to chest infections in these patients. The authors conducted a pilot study, analysing retrospective data to explore this observation further.

Methods All variant AT patients who attended a multi-disciplinary (MDT) annual review appointment between 01/2013$11 / 2015$ and underwent a videofluoroscopy were included. The patient was ranked on the Penetration Aspiration Score (PAS) from the videofluoroscopy. Retrospective data of the frequency of chest infections requiring treatment with antibiotics in the last 12 months was collected from the annual assessment reports in the medical notes.

Initially, 37 patients were included, this was reduced to 30 as 7 videofluoroscopies were not sufficient for calculating the swallow trigger delay. Data was analysed using excel and xlstat (standard descriptive tests and Spearman's) to evaluate any correlation between aspiration or penetration and number of chest infections.

Results With a confidence interval of $95 \%$, there was a negative correlation of rs 0.079 ( $\mathrm{p}$-value $<0.0001$ ) indicating a very weak correlation. The number of chest infections data was significantly skewed at a result of 2.643 . This may be due to the small sample size.

Conclusions This data suggests there is no correlation between aspiration or penetration and the chest infections experienced by AT Patients. However, this was a small sample due to the rarity of the genetic disorder. A larger sample either through increased length of time that the data is collected or an international study could help provide greater insights into the correlation between aspiration or penetration of oral intake and the number of chest infections experienced.

\section{REFERENCE}

1 Rosenbek JC, Robbins JA, Roeker EB et al. A penetration-aspiration scale. Dysphagia 1996;11(2):93-98.

\section{P263 DOES THE USE OF LACTATE IMPROVE THE CURB-65 SCORE IN COMMUNITY ACQUIRED PNEUMONIA PATIENTS ADMITTED TO A DISTRICT GENERAL HOSPITAL?}

TW Nicholson, S Connaire, V Kronsten, S Black, JES Park. Department of Respiratory Medicine, Royal Berkshire Hospital, Reading, UK

10.1136/thoraxjnl-2016-209333.406 


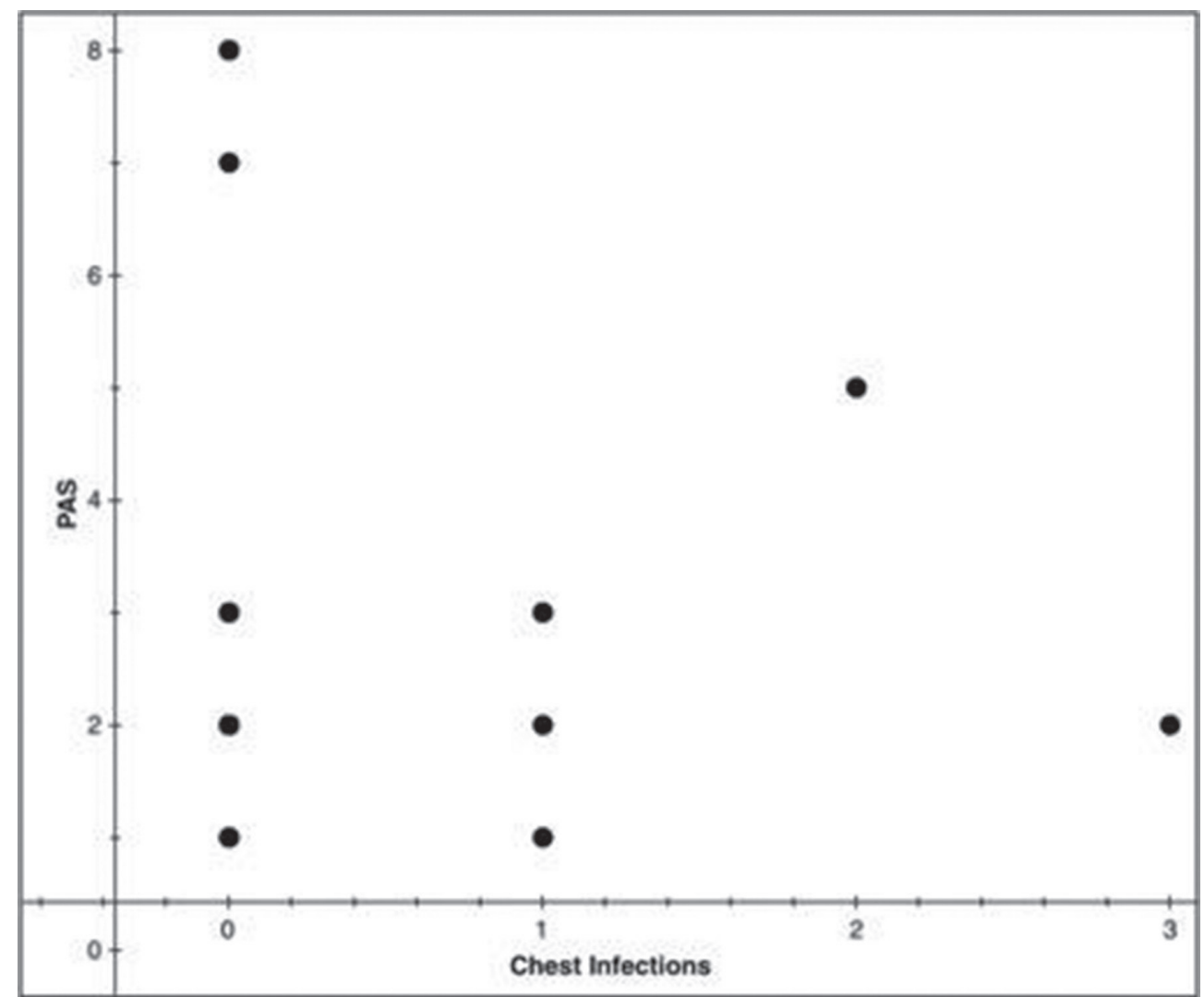

Abstract P262 Figure 1 Aspiration penetration and number of chest infestions

Community acquired pneumonia (CAP) is a leading cause of admission and mortality. CURB-65 is the traditional risk stratification score. This can under predict severity in the young and does not predict requirement for higher level care. Combining CURB-65 with lactate can improve this. ${ }^{1}$ We reviewed CAP admissions over one month to determine if lactate improved risk stratification.

CAP patients were identified via coding. Authors reviewed admission chest radiographs and reports to confirm CAP. CURB65 score was calculated from the electronic patient record (EPR), and electronic discharge letters. Lactate values were identified from Emergency Department documents scanned into EPR. Accuracy of coding and mortality in a wrongly coded group was a secondary measurement. A LAC-CURB score of low, medium or high was allocated.

138 episodes of CAP were coded. 89 were confirmed CAP. Mean age was 71.2 yrs (21-98). CURB-65 score was available in 87. 45 scored CURB-65 0-1 with 2 deaths (4.4\%). 24 were CURB-65 2, with 8 deaths (33.3\%) and 6 of 18 CURB-65 3-5 patients died (33.3\%).

A lactate value was available in 52. 16 had a low LAC-CURB score with 0 deaths and 1 ICU admission. 23 had a medium score, with 3 deaths (13\%) and 2 ICU admissions. 13 had a high LAC-CURB score, with 4 deaths (31\%) and 1 ICU admission. In 4 patients it was the lactate value that increased the risk category from a medium CURB-65 score to a High LAC-CURB score. All 4 were admitted to ICU, with 2 deaths. Length of stay did not alter significantly with CURB-65 or LAC-CURB, but increased with severity. Diagnoses were available in 45 of the 49 patients coded incorrectly as CAP with a $17.78 \%$ mortality rate, identical to the CAP group.

Mortality was higher for medium and high CURB-65 patients, but a difference between them was only seen when the LACCURB score was applied. A high lactate identified patients in the medium CURB-65 group who died or required higher level care. Patients wrongly coded as CAP also have a high mortality.

\section{REFERENCE}

1 Chen YX. Thorax 2015;70(5).

\section{P264 PREDICTING MORTALITY IN HOSPITAL ACQUIRED PNEUMONIA: A MULTIVARIATE ANALYSIS}

${ }^{1} \mathrm{CB}$ Morris, ${ }^{2} \mathrm{~S}$ Valapraya, ${ }^{1} \mathrm{E}$ McCance, ${ }^{1} \mathrm{AM}$ Turner, ${ }^{2} \mathrm{D}$ Dosanjh. ${ }^{1}$ Heart of England NHS Foundation Trust, Birmingham, UK; ${ }^{2}$ University of Birmingham, Birmingham, UK

\subsection{6/thoraxjnl-2016-209333.407}

Background Hospital Acquired Pneumonia (HAP) is defined as lung infection in a non-intubated patient with new infiltrates on chest X-ray, $>48$ hours after hospital admission. Prediction scores exist for Community Acquired Pneumonia (CAP); no such scores exist in HAP. We aimed to identify features which are predictive of mortality in HAP.

Methods All cases coded as HAP in Heart of England Foundation NHS trust in 2013 trust were identified (293 cases). For each of these cases the chest X-ray (including radiologists report) was reviewed; if X-ray did not show infiltrates consistent with pneumonia, cases were excluded leaving 153 cases for whom case notes were reviewed. Cases were excluded if diagnosis of HAP 